\title{
A Metacognition Based 8 Pillars Mindfulness Model and Training Strategies
}

$\underline{\text { https://doi.org/10.3991/ijes.v8i4.17419 }}$

\author{
Athanasios Drigas ${ }^{(凶)}$, Eleni Mitsea \\ Net Media Lab-Mind \& Brain R\&D, \\ N.C.S.R. 'Demokritos', Athens, Greece \\ drait.demokritos.gr
}

\begin{abstract}
Oxidative stress, mental and mood disorders, premature ageing, cognitive impairments, learning disabilities are just some of the emerging threats of the 21 st century. The next generation of educators, students, business leaders, employees, therapists, and parents are required to adapt in an era of rapid change and climate change by developing new skills as well as adopting daily sustainable habits. An increasing number of studies reveal the positive effects of mindfulness training in all dimensions of human existence (bodily, emotionally, mentally, and spiritually). Nevertheless, there is a research gap regarding the pillars of mindfulness. The present work proposes a mindfulness model based on the potential principles and the mechanisms of metacognition. Adopting a holistic self-development view, we present the fundamental pillars of mindfulness and their underlying operations. Each pillar entails a number of techniques that help to cultivate certain metacognitive abilities and qualities, leading gradually to the higher levels of self-organization, intelligence and consciousness. We highlight the positive effects of mindfulness training on antiageing, stress reduction, neuroplasticity, brain rewiring and hormonal homeostasis.
\end{abstract}

Keywords-Attention, consciousness, self-awareness, executive functions, self-regulation, self-observation, adaptation, neurotransmitters, hormones, stress reduction, brain rewiring, self-fulfillment, special education

\section{Introduction}

According to the World Health Organization, stress constitutes the "health epidemic" of the 21st century. In the last twenty years, mindfulness is one of the most popular topics in psychology, psychiatry, medicine and neuroscience [1]. Mindfulness training is promoted as a novel method in leadership development since many researchers explore how mindfulness training may affect leading capabilities [2]. Mindfulness practices are key factor in personal and professional development. In many schools of general and special education, mindfulness-based programs are adopted as valuable tools for bodily, cognitive, emotional as well as social improvement [3]. Meditation practices are among the most popular therapeutic activities that involve 
physiological, psychological and neurological processes resulting in relaxation, stress reduction and the improvement of mental and physical health [4]. Yet, various physical or mental activities (e.g. martial arts, balance training, digital games) are used effectively as alternative methods of practicing mindfulness skills [5-7].

Despite the multitude of research, the mechanisms and the functions that underlie mindfulness remain unclear. In the current study, we propose a mindfulness model that classifies and describes the metacognitive mechanisms of mindfulness, taking into account the relevant physical, emotional and mental operations. The aforementioned operations are treated both as tools for cultivating superior metacognitive abilities and as objects of continuous improvement. The various practices are classified according to the demands of each pillar, demonstrating that mindfulness practices may in fact sustain specific skills.

\section{A Mindfulness Model Based on Metacognition: The 8 Pillars of Mindfulness and Training Strategies}

\subsection{First pillar: Knowledge of what are the components of our existence bodily, emotionally, mentally and spiritually}

The concept of mindfulness is tied to a dynamic and unbroken, through the centuries, theoretical background about the human existence as a unity of bodily, emotional, mental and spiritual forces. Thus, the first step in approaching the concept of "mindfulness" requires a holistic knowledge and deep understanding of ancient and contemporary theories that are related to each part of human existence. This knowledge can be structured in two central axes the physiological and the cognitive.

One of the oldest traditional systems of medicine, Ayurveda underlines that health is about balance between the outside world and the fundamental states of "being" namely the physical (including the physiological), the mental and the spiritual. Specifically, it implies stability between structural and physiological factors, metabolic and excretory processes, body tissues, the senses, the mind and the attainment of a state of self-awareness [8]. These processes are understood according to the theory of five elements (earth, water, fire, air, space) which correspond to each of the five senses (smell, taste, vision, touch and sound) and are further grouped into three energies called tridosa (vata, pitta and kapha) [9].

Indubitably, knowledge of the endocrine system, which is of several glands, is more than essential. The thalamus, the hypothalamus, the pituitary gland, the pineal gland, the thyroid and parathyroid glands, the thymus, the adrenal glands, the pancreas and the gonads produce hormones that regulate many of the bodily functions, influencing mental health and well-being [10]. Neurotransmitters are crucial to the functions of the central nervous system including perception, memory, alertness, sleep cycle, body movement, energy, behavior and hormone regulation. The brain sends neurotransmitters to tell the heart to beat, the lungs to breathe and the stomach to digest. The inhibitory neurotransmitters calm the brain while they are easily depleted when the excitatory neurotransmitters are overactive. The most important inhibitory 
neurotransmitters are acetylcholine, norepinephrine, dopamine, GABA, glutamate, serotonin and endorphins. The function of all such chemical elements presupposes scientific knowledge regarding the proper supply of the body with appropriate vitamins, minerals and trace elements that contribute to fundamental functions such as the synthesis of neurotransmitters [11]. One should be knowledgeable about environmental toxicity (e.g. chemicals in genetically modified foods, pesticides, heavy metals) that harms the immune system and cognitive ability, degrade intelligence, alter human behavior and cause learning disabilities [12].

Amongst others, neurotransmitters influence brain areas such as the prefrontal cortex, the amygdala, the hippocampus and many other areas crucial for metacognition, intelligence and consciousness [13-15]. The hippocampus, for instance, is orchestrated with the prefrontal cortex, the amygdala and the hypothalamus to generate attention, memory and imagery as well as emotional processing that permit one to recognize, discriminate, understand and channel one's negative emotions to enhance the positive ones [16-18].

Mindfulness is closely associated with brain areas like the prefrontal cortex that is the foundation of executive functions. These functions offer a physical substrate of intelligence and encompass superior cognitive abilities like attention, working memory, cognitive and emotional regulation, cognitive flexibility, inhibition control, organization, reasoning, problem solving, decision making and concept formation. All of the above facilitate the flexible modification of thought and behavior in the face of novel demands [3,19]. Thus, theoretical instruction should focus on theories of intelligence and the most fundamental cognitive processes (attention, memory, perception, association, concept formation, pattern recognition, language, action, problem solving and mental imagery) through which we can collect, synthesize and transform data to information, and information to knowledge [13,17]. These cognitive functions permit us to analyze, to categorize, to associate information, to uncover relations between concepts, to draw connections between objects and events, to extrapolate beyond the given information. On the spiritual level, we must engage with the theories of spirituality, which underline the role of the real self, the deep motives, ethical development, the ultimate potentialities, the holistic levels of human consciousness [18].

\subsection{Second pillar: Knowledge of what is the usual behavior of the components of our existence. Knowledge of a SWOT analysis (strengths, weaknesses, opportunities and threats) of our situation}

Mindfulness is not achieved simply by a theoretical approach; On the contrary, we must apply theory in real life so as to gain awareness of every situation. For instance, without awareness or control of basic cognitive processes such as attention and working memory, we are unable to select and maintain information at a given moment resulting inevitably in mind wandering away from the present moment to taskunrelated thoughts and feelings. [20]. When we practice mindfulness, we "open" the gateway to awareness, since more attentional resources are available to process information relative to the current experience. Perceptual ability and access to information expands. [19]. Visualization-based meditation involves crucial sub-systems of 
the working memory such as the visuospatial sketchpad, resulting in mental imagery enhancement. Moreover, the aforementioned practices by reducing activation of language areas have an indirect impact on emotional regulation. [21].

Awareness includes knowledge of the real factors/causes (e.g. environmental, nutritional, endocrinological, immunological, and psychological) that accelerate or slow down the physical, emotional, mental and spiritual processes. Nutritional factors, either positive or negative, affect cognitive abilities and modify the entire brain [12].

Uncontrollable stress often disrupts homeostasis [4] leading to mind wandering, engagement, and rejection of the present moment; while positive social interaction enhances presence in the moment [22]. On the other hand, meditation practices are associated with neuroplasticity phenomena, help in reducing age-related brain degeneration and improving cognitive functions [23].

Mindfulness is about our awareness of strengths and weaknesses. Usually, we tend to associate emotionally charged feelings with seemingly automatic responses. Like any other behavior, such feelings are learned and thus can be unlearned through reevaluation of an initial reaction to a certain stimulus and modify it so as to achieve objectivity, positivity, acceptance and self-actualization. Training in mindfulness strategies has been proven to be effective in turning negative emotions into positive ones [24].

2.3 Third pillar: Self-monitoring and watching various emotional and mental movements in a real time framework. We construct an internal observer. We rest in observation. We observe the thoughts, the emotions, the stresses, the relaxation, the quietness and serenity among noises, the void among thoughts. We observe the observer

In a state of awareness, we notice sensations, thoughts and feelings in a reflective way, as ever-changing events or movements in the mind, as "objects" of observation without over-identifying with them, without reacting to them in an automatic habitual pattern of reactivity. This observant stance towards current experience could be compared to a "tracking system", a "radar" that uses, for instance, the sustained attention on the breath so as to detect mental and emotional movements as they arise in the stream of consciousness $[19,25]$. However, when distractors such as beliefs, assumptions, expectations and desires get in the middle, the "radar's" ability to detect and identify stimuli or objects in unexpected contexts decreases. By applying Body Scan practice, we obtain a more accurate perception of sensory reality, direct attention at different areas of the body, by noticing any sensation or muscle tension in each area. By this exercise, we improve our self-awareness, the ability to understand complex mental and emotional states, distinguishing different elements of experience such as signals from sensory "noise", cognitive from emotional experiences, feelings from bodily sensations [26]. Self-observation is achieved gradually through various meditation techniques such as walking or lying meditation and sitting in silence. In walking meditation, we observe senses (movement of body, clothes, air, hair) coordinating movement with breath if necessary. Lying meditation involves lying down in a state of deep relaxation while being fully conscious. A lot of people have difficulty sitting 
still and do nothing but observe the quietness and serenity that surrounds them. Sitting in silence voluntarily is associated with an increase in observance and awareness, especially in terms of inner experience. This practice has helped undergraduate students to reduce their anxiety and impulsivity [1]. In the dispassionate state of selfobservation, we recognize the "space" between thoughts which allows the discovery of new ways of perceiving and responding. It is not about thoughts but rather the "space" between thoughts where mindful individuals experience the arising "self" as a process of integration $[18,19]$.

\subsection{Fourth pillar of self-regulation: The observation procedure brings regulation, eases of thoughts and emotions, relaxation deepens and observation becomes clearer}

Mindful self-regulation is one of the most crucial mechanisms whose gears, namely the executive functions relying on the prefrontal cortex, enable us to attain and maintain a state of presence, awareness and consciousness without mind wandering, suppression or defensive attitude over thoughts and emotions. Self-regulation training helps us identify any current thoughts, feelings, perceptions, behavior and thus try to manage them in real time. [3,19,27]. Attentional control processes constitute the heart of the executive functions and include certain fundamental abilities that culminate in a state of mindfulness. Specifically, the aforementioned processes include the abilities to allocate attention towards internal and external stimuli in a most plausible way, to reach and maintain a state of vigilance, to focus attention for a prolonged period to visuo-spatial stimuli, to be flexible, to prioritize [28]. Attentional control is a prerequisite for emotional regulation as well as emotional awareness. Specifically, it means improvement of the emotional regulation processes since we learn pay attention to emotional responses, filtering emotional states and taking into consideration all possible aspects of emotional experience [29]. Highly mindful individuals are said to be "in tune" with their emotions and capable of regulating them [25]. A further aspect of self-regulation underlines the contribution of language. Mindful individuals use less negative word meanings because of successful self-regulation of attention and emotion. Also, visualization-based practices improve the ability to self-regulate, as they require less language involvement [27].

Breathing techniques and relaxation treatments are superior methods that regulate negative mood states such as stress, anxiety and depression in a holistic way. The respiratory activity plays a significant role in the autonomic nervous system, cardiac activity, homeostatic changes and emotions. Slow, deep breathing and cardiorespiratory synchronization may lead to homeostatic increase in cellular membrane potentials and a generalized decrease in excitability of heart and the amygdala [30]. Body movement boosts attention, self-control and mindfulness. Considering the Attention Deficit/Hyperactivity disorder in which deficient motor control correlates with disinhibited behavioral control results in excessive distractibility and impulsivity. It is obvious that there is a common basis between executive and sensorimotor control functions. Mind and body training like qigong, aikido, tai chi, judo, dance, yoga sustain one's ability to monitor movement in a flexible way maintaining attention within 
the movement. Consequently, such techniques improve both the prefrontal and the premotor cortex [31]. Tai Chi and Qigong promote coordinated body posture and movements, deep rhythmic breathing, meditation and mental focus, enhancing no reactivity to aversive thoughts and impulses [32]. The slow movements of Qigong and Tai chi alter the autonomic nervous system; they restore homeostasis by attenuating stress, related to the hypothalamus-pituitary-adrenal axis reactivity. Additionally, researches show improved functionality and connectivity within multiple brain regions involved in mood regulation including prefrontal regions, limbic systems, motor cortex and striatum. Tai chi reduces neuro-inflammatory sensitization and regulates the hippocampal neurogenesis, resulting in increased attentional and emotional control and self-awareness [7].

Eating is often an impulsive reaction, an emotional coping strategy so as to escape negative emotions. Mindfulness eating exercises (e.g. to eat in silence, eat slowly, and notice we are full, stop eating when we are full, stop mind wandering while eating) constitute beneficial regulatory strategies [33].

2.5 Fifth pillar of adaptation: The body, emotional and mental operations become more adaptable, more relaxed, while breathing operates smoothly, relaxed and adapted to the present moment. Perception deepens and antistress hormones are secreted as well as neurotransmitters supporting relaxed awareness

Various forms of meditation like guided meditation, deep relaxation and breathing techniques bring homeostatic balance throughout the body, by ways of reducing the amygdala's activity, the stress hormones' secretion (e.g. cortisol, adrenaline), releasing chemicals that bring relaxation and optimism (e.g. endorphins, serotonin), regulating blood pressure, muscle tension, body temperature, oxygen consumption. During the aforementioned techniques, neurotransmitters such as gamma-aminobutyric acid induce relaxation facilitating well-tempered activation and communication between the cortex and the amygdala as well as respiration with cardiac centers. In addition, the alpha waves of relaxation are increased, while beta waves are decreased. Existing neural pathways and circuits become permeable, while new neurons and connections are formed. Subsequently, body and mental stress symptoms or negative emotions smooth over in a parallel with muscle tensions. Moreover, attentional resources are available to process any information relative to a current experience. By regulating attention, irrelevant thoughts and negative emotions decrease. The working memory sub-systems gain valuable space and as a result memory gets to function more smoothly, efficiently. [3,19,30].

Behavioral flexibility permits us to generate, update, modify and integrate past and present information in response to any demands and constraints of a real life environment. The hippocampus underlies conscious cognition as well as flexible social behavior permitting us to remember, explore, visualize, create, make decisions, judge character objectively, establish social bonds, feel empathy, and speak the social language [34]. Adaptability is increased by learning or training spatial memory via balance exercises which bring change in the hippocampus. In the same way coordinative 
training, aerobic exercise, stretching as well as relaxation techniques such as progressive muscle relaxation could contribute in adaptation skills improvement [5].

Adaptability skills equal the mindfulness skills of the 21 st century since they are a prerequisite for adapting successfully to dynamic team environments (educational, academic or working). Such environments require rapid, adaptable and constantly focused to objective response to unexpected changes. Rupprecht et al. [2] found that leaders in major organizations that completed a 10 -week workplace mindful training (including mindful meditation, mindful listening, walking meditation, pausing meditation, body scan and compassion meditation) improved their ability deal with change and focus on giving alternative and creative solutions to given problems. Flexibility and adaptability lead to active self-observation, self-regulation of attention and emotional self-control. Adaptability, as a mindfulness trait involves increased social and emotional skills, resilience, positive thinking, open-minded and active listening, problem solving, decision-making, innovation, creative strategy thinking skills.

Mindless eating results in various problems (body, emotional, mental, social even ecological). Adaptation implies also the cultivation of conscious and healthy eating behaviors, along with an effort to protect the environment and animal welfare. Mindful eating increases self-reflexion by thinking about the origin of the food, about "what we eat", "how much", "how", and "why we eat". In the context of mindfulness, adaptation implies consuming less animal products. [33].

\subsection{Sixth pillar of recognition: Expansion of awareness and perception bring recognition of the details as well as of the totality of each situation. We perceive multiplicity and unity in parallel.}

Recognition is a state of heightened awareness in the present moment regardless of circumstances, resulting from the ability to broaden our perceptual field through a process of synchronization between the internal world of mental events (i.e. automatic thoughts, ineffective strategies, emotions, beliefs, strengths, weaknesses and motives) and the external world of objects via the five senses. It has an integrative function since it tends to unify all experience, internal and external bringing their profound relationships to the "light" of consciousness [17-18]. The practice of attending and mindfully observing to body sensations results in an enhanced awareness of bodily states and reinforced perceptual clarity. Increased body awareness leads to greater sensitivity to detect the physiological aspects of any feelings of the moment (body, tension, rapid heartbeat, short shallow breath) which in turn implies enhanced emotional recognition, awareness and empathy [35]. Mindfulness eating helps us to recognize when food advertisements make us want to eat, to recognize when we are eating without being hungry [33].

During this process of self-understanding, we reflect on thoughts approaching them as an outcome of internal and perhaps unconscious processes while triggered also by external environmental stimuli. It is not enough to identify stress or negative emotions such as anger; instead, we have to recognize the possible dimensions and the real causes that provoke them. In other words, recognition is about the ability to become aware of what causes, for instance, stress in a given situation. A real danger? A hypo- 
thetical danger? Our illusive expectations, our desires, our attachment to the phenomenological world, or biological factors like a disharmony of homeostasis? As we reache a higher level of understanding, we recognize thoughts and feelings as symptoms, as indicators of deeper pathogenesis of somatic or/and psycho-cognitive etiology. If we are not able to observe and recognize the phenomena and their substrate, it is not possible to mobilize the appropriate self-regulatory strategies. Only if we recognize and accept our emotions with openness, only with empathy, are we able to manage them appropriately. As we train our observation skills, we improve the ability to identify the underlying structure of sensory, emotional and mental phenomena, operations, their relations and correlations $[17,36]$.

As attention strengthens through nutrition, physical or mental exercise, the range of observation expands. We observe consciously and in detail not only the thoughts that follow each other as well as the space that is the ground of existence, including ourselves. It is within the space that consciousness and giftedness arise, manifesting initially as a glimpse of higher-order beliefs, self-actualizing intentions, gratitude, ideas, original and innovative, the realization of the interconnectedness of all ideas $[13,17$ 19]. In addition, we recognize our capacity to transcend limitations and identify with a universal non-personal awareness [37].

According to Batchelor [38], "normally we are unaware of the extent to which we are distracted, for the simple reason that distraction is a state of unawareness. Breathing exercises can force us to recognize that most of the time, we fail to register what is happening here and now". In open monitoring practices, we cultivate, through bottom-up processes, mainly the ability to expand our attention to the flow of energy (sensory as well as psychocognitive) with the aim to conceive a panoramic "view" of the totality that exists behind every singleton experience with a mood towards acceptance rather than control.

2.7 Seventh pillar of discrimination: In this open recognition state, we distinguish the various forms, movements, states and representations. We keep beneficial and withdraw attention from negative so that the negative ceases to exist in our mental field.

Mindfulness not only registers the bare facts of internal and external experiences but also introduces the element of discernment, enabling one to evaluate whether a particular experience nourishes the "seeds" of positive mental states (e.g. constructive emotions, loving-kindness) or conduces to destructive ones (i.e. anger, desirous attachment, lethargy). Thus, we train our attention so as to notice subtle differences between emotional experiences [24,29] and move away from "toxic" and harmful states of mind and thus avoid inappropriate behaviors and actions. Discrimination executes its filtering function without suppression or reactive rejection. In contrast, discrimination abilities arise in a state of tranquility and stillness of mind, when attention is stabilized [39]. We can compare the human mind with a lake and thoughts to a stone tossed into water. When the lake is still the ripple is clearly visible, otherwise, it is barely noticeable [40] (Bodhi, 2015 as cited in Vago, 2016). If one holds a glass of muddy water still, the dirt will settle to the bottom and the water will shine. A highly 
developed meta-awareness in the context of mindfulness-based practices leads to rapid discernment of what is relevant from the early stages of attentional processing. In addition, it provides sensory clarity and emotional stability [40]. Discrimination does not promote rejection but willingness to transform destructive states and tendencies into constructive thoughts, emotions and purposeful actions [39].

Intensive meditation that involves sustained attention on a chosen stimulus (e.g., the participant's breath) improves visual sensitivity and discrimination, vigilance and perception. Moreover, mental training on nonvisual perceptions (e.g. sensations of breathing and mental events) improves visual perception. [41] Walking meditation could foster auditory and visual discrimination skills. The Transcendental Meditation (TM) technique as one sits in a chair with one's eyes closed. During TM instruction, the individual learns how to let the mind move from active focused levels of thinking to silent, expanded levels of wakefulness. Loving-kindness meditation and compassion meditation exercises aim at enhancing kindness and compassion. These techniques broaden attention, enhance positive emotions, and lessen negative emotional states; they shift a person's basic view of the Self in relation to others and increase empathy and compassion [27,42]. Digital games or other discrimination tasks (as to language, STEM and mathematics) could contribute to the acquisition of mindful discrimination skills $[6,12]$.

Discrimination skills could be cultivated through strategic management training. Top managers must assess the similarities as well as the differences between current and past decision-making situations in order to choose appropriate behaviors or actions for present or future situations. These skills involve the ability to retrieve from memory, to segment actions to situations, to recognize the salience of the current situation, to do a comparative assessment, to make conscious and appropriate choices, to transform the experience to useful knowledge [43].

2.8 Eighth pillar of Mnemosyne: The state of awakening and full awareness. Mnemosyne supports and drives all the mindful procedures, keeps us in an aware state, reminds to us of the real existential dimensions, the real self and its capabilities and finally reminds us of the return road of anelixis to our real existential level.

A seed "knows" its potential because it is gifted with the necessary information to grow into a "real" plant. We have a "memory" within us, a huge and hidden potential that waits to be remembered, awakened, actualized and transcended. Mnemosyne is the awakening of consciousness. The path to the hidden potential is nothing more than the ascending path of anelixis to the higher levels of self-awareness and consciousness. This journey on the road to anelixis is a return journey during which we must sail against the entropy flow, defeat the delusions that prevent us from "seeing" our true existential dimension. To achieve this, we must remember at every moment that the knowledge we strive to acquire is not spoils but a compass, a reminder of our true destination. We must keep in mind that knowledge is acquired through continuous transformation, after which the newly acquired knowledge is never the same as any previous one; the perception of the new self is not the same as any previous one. The 
ascent on the ladder of knowledge goes hand in hand with the improvement of intelligence and emergence of consciousness. The Self, its various forms and transformations is nothing more than a manifestation of consciousness at different levels of self-remembrance [13, 17-18].

Practices that induce a perceptual shift away from egoistical self-identification could make one "grow", achieving a state of self-fulfillment that leads to optimal wellbeing. More specifically, we could mention contemplation of the simple question "who am I", sitting in open awareness without any specific object of focus, minimizing phenomenal content via the use of mantra or focused attention, or increasing contact with the internal space of the body [37].

A mindful person remembers to learn, to take care and train the mind as well as the body, to be attentive to the present moment, to observe internally and externally, to be alert in order to discern and recognize different situations, operations, correlations, to self-regulate, to adapt, to accept, to concentrate on his true existential nature.

\section{Discussion}

Any practice that fosters the mindful skills like meditation, yoga, martial arts as well as various physical or mental exercises is a way to boost new neurons' growth (neurogenesis) and also to form new connections between existing neurons (synaptogenesis) [44]. Neural pathways and circuits become more fordable, upgrading cognitive functions as well as metacognitive abilities, resulting in major improvements in terms of monitoring, regulating and adapting in stress-related situations. Mindfulness is a process of brain rewiring, since we are required to replace dysfunctional established habits with new, more useful and functional [17]. Mindful practices contribute to improving our intelligence, and self-awareness in a variety of way. One such way includes maintenance of hormones' and neurotransmitters' homeostasis, a regulation of the interaction between the hypothalamic-pituitary- adrenal axis, the central as well as the autonomic nervous system, the limbic system and the immune system [44]. Mahajan [45] reviewed some of the beneficial effects of yoga and other meditation techniques on stress related hormones/neurotransmitters. Specifically, there is a decline in adrenaline and cortisol levels. Practitioners experience joy and euphoria because of the increase of b-endorphins, serotonin, dehydroepiandrosterone sulfate and dopamine levels. Also, melatonin levels increase, giving a feeling of calmness and well-being. Krishnakumar et al. [44] refer to the increase of gamma aminobutyric acid that is associated prefrontal cortex activity that in turn influences the executive functions, concentration and alertness. Moreover, lower levels of norepinephrine in meditators indicate lesser anxiety. Although different techniques may produce different results, it is obvious that mindfulness and its practices have positive effects on brain rewiring, hormonal homeostasis, anti-aging and anti-stress functions [45]. Obviously, results are optimized when there is proper monitoring, regulation and adaptation of one's nutritional behavior. A healthy diet detoxifies the body allowing homeostasis to function properly $[9,12]$. 
Mindfulness practices are at the heart of attention and control. Every time we train our attention, we improve awareness, we enhance self-control as well as the pathways of memory that are responsible to control. So, the "gates" open for improvement of other dependent operations and abilities on a physical, emotional, mental and spiritual level.

Cultivation of mindfulness has beneficial effects on well-being, while it alleviates stress-related symptoms [35]. Conventional psychopharmacology and behavioral therapies are essential. Nevertheless, we can achieve more if we integrate mindfulness within these treatments. Health professionals and educators have received limited education concerning such interventions. Thus, they may be unaware of mindfulness training benefits or the way mindfulness works. Additionally, mindfulness training has been proven an efficient, low risk and low-cost intervention for patients and students. Education in the $21^{\text {st }}$ century ought to aim at developing competent students and adults in all aspects of Life, armed with a good set of skills including mindfulness. So that the young people may improve their physical, emotional, intellectual as well as spiritual intelligence, adopt healthier habits; be more attentive and conscious, motivated, less anxious, self-controlled and adaptable. Moreover, they will also have a better chance at career opportunities.

\section{Conclusion}

On a theoretical and practical level, mindfulness encapsulates the fundamental mechanisms of metacognition and consciousness. In other words, metacognition and mindfulness are the two opposite sides of the same coin. Mindfulness training leads to metacognitive improvements and metacognitive training results in a state of mindfulness. Metacognition and mindfulness depend on our ability to monitor, control and adapt our thoughts and emotions, recognizing and discriminating between functional and dysfunctional mental or emotional states in order to get to know the full range of our abilities, who we really are.

Summarizing the fundamental pillars of mindfulness:

1. Holistic theoretical knowledge and deep understanding of the mechanisms, the underlying operations as well as the factors that contribute to the proper function of our existence as a unity of bodily, emotional, intellectual as well as spiritual power.

2. The application of the theories in real life, so as to be aware of our physical, emotional, intellectual and spiritual power, to understand and evaluate the range of our strengths and weaknesses.

3. The cultivation of a vigilant observant stance, both internal and external, toward the current experience.

4. Self-observation and self-control processes regulate thought, emotional response and behavior resulting in homeostatic balance.

5. Adaptation of bodily, emotional and mental operations in a climate of positiveness, altruism and acceptance.

6. Recognition of the details as well as of the totality of each situation with a mood of openness, gratitude and empathy. 
7. The development of discrimination skills helps us to observe, filter and choose the beneficial instead of toxic states.

8. Mnemosyne is a state of full awareness by which the internalized knowledge reminds us the return road of anelixis, the evolutionary process of self-development to awaken the higher levels of consciousness.

\section{$5 \quad$ References}

[1] Matko, K., \& Sedlmeier, P. (2019). What is Meditation? Proposing an EmpiricallyDerived Classification System. Frontiers in psychology, 10, 2276. https://doi.org/10.3389/ fpsyg.2019.02276

[2] Rupprecht, S., Falke, P., Kohls, N., Tamdjidi, C., Wittmann, M., \& Kersemaekers, W. (2019). Mindful leader development: how leaders experience the effects of mindfulness training on leader capabilities. Frontiers in psychology, 10, 1081. https://doi.org/10.3389/ fpsyg.2019.01081

[3] Drigas, A., \& Karyotaki, M. (2018). Mindfulness Training \& Assessment and Intelligence. International Journal of Recent Contributions from Engineering, Science \& IT (iJES), 6(3), 70-85. https://doi.org/10.3991/ijes.v6i3.9248

[4] Pascoe, M. C., Thompson, D. R., \& Ski, C. F. (2020). Meditation and Endocrine Health and Wellbeing. Trends in Endocrinology \& Metabolism. https://doi.org/10.1016/j.tem. 2020.01 .012

[5] Rogge, A. K., Röder, B., Zech, A., Nagel, V., Hollander, K., Braumann, K. M., \& Hötting, K. (2017). Balance training improves memory and spatial cognition in healthy adults. Scientific reports, 7(1), 1-10. https://doi.org/10.1038/s41598-017-06071-9

[6] Donohue, S. E., Woldorff, M. G., \& Mitroff, S. R. (2010). Video game players show more precise multisensory temporal processing abilities. Attention, perception, \& psychophysics, 72(4), 1120-1129. https://doi.org/10.3758/app.72.4.1120

[7] Kong, J., Wilson, G., Park, J., Pereira, K., Walpole, C., \& Yeung, A. (2019). Treating depression with Tai Chi: state of the art and future perspectives. Frontiers in psychiatry, 10, 237. https://doi.org/10.3389/fpsyt.2019.00237

[8] Payyappallimana U, Venkatasubramanian P. In: Verotta L, Pia Macchi M, Venkatasubramanian P, editors. Principles of Ayurveda for Food, Nutrition, and Health in Connecting Indian Wisdom and Western Science - Plant Usage for Nutrition and Health. New York: CRC Press (2015). p. 15-36. https://doi.org/10.1201/b18430-4

[9] Payyappallimana, U., \& Venkatasubramanian, P. (2016). Exploring Ayurvedic knowledge on food and health for providing innovative solutions to contemporary healthcare. Frontiers in public health, 4, 57. https://doi.org/10.3389/fpubh.2016.00057

[10] Glaser, R., \& Kiecolt-Glaser, J. K. (2005). Stress-induced immune dysfunction: implications for health. Nature Reviews Immunology, 5(3), 243-251. https://doi.org/10.1038/ nri1571

[11] Gibson, G. E., Blass, J. P., Siegel, G. J., Agranoff, B. W., \& Albers, R. W. (1999). Nutrition and functional neurochemistry. Basic Neurochemistry: Molecular, Cellular and Medical Aspects. 6th ed. Philadelphia: Lippincott-Raven, 691-710.

[12] Chapin, T. J., \& Russell-Chapin, L. A. (2013). Neurotherapy and neurofeedback: Brainbased treatment for psychological and behavioral problems. Routledge. https://oi.org/ $10.4324 / 9780203072523$

[13] Drigas, A. S., \& Pappas, M. A. (2017). The Consciousness-Intelligence-Knowledge Pyramid: An 8x8 Layer Model. International Journal of Recent Contributions from Engineering, Science \& IT (iJES), 5(3), 14-25. https://doi.org/10.3991/ijes.v5i3.7680 
[14] Myhrer, T. (2003). Neurotransmitter systems involved in learning and memory in the rat: a meta-analysis based on studies of four behavioral tasks. Brain Research Reviews,41(2-3), 268-287. https://doi.org/10.1016/s0165-0173(02)00268-0

[15] Sasikumar, N. INFLUENCE OF NEUROTRANSMITTERS ON MEMORY AND LEARNING.

[16] Deshmukh, V. D. (2006). Neuroscience of meditation. The Scientific World Journal, 6, 2239-2253.

[17] Drigas, A., Mitsea E. (in press). The 8 pillars of Metacognition. International Journal of Emerging Technologies in Learning (iJET)

[18] Drigas, A., \& Mitsea, E. (2020). The Triangle of Spiritual Intelligence, Metacognition and Consciousness. International Journal of Recent Contributions from Engineering, Science \& IT (iJES), 8(1), 4-23. https://doi.org/10.3991/ijes.v8i1.12503

[19] Bishop, S. R., Lau, M., Shapiro, S., Carlson, L., Anderson, N. D., Carmody, J. \& Devins, G. (2004). Mindfulness: A proposed operational definition. Clinical psychology: Science and practice, 11(3), 230-241. https://doi.org/10.1093/clipsy.bph077

[20] Morrison, A. B., \& Jha, A. P. (2015). Mindfulness, attention, and working memory. In Handbook of mindfulness and self-regulation (pp. 33-45). Springer, New York, NY. https://doi.org/10.1007/978-1-4939-2263-5 4

[21] Kozhevnikov, M., Louchakova, O., Josipovic, Z., \& Motes, M. A. (2009). The enhancement of visuospatial processing efficiency through Buddhist deity meditation. Psychological Science, 20(5), 645-653. https://doi.org/10.1111/j.1467-9280.2009.02345.x

[22] Crosswell, A. D., Coccia, M., \& Epel, E. S. (2019). Mind wandering and stress: When you don't like the present moment. Emotion. https://doi.org/10.1037/emo0000548

[23] Lardone, A., Liparoti, M., Sorrentino, P., Rucco, R., Jacini, F., Polverino, A., \& Ferraioli, G. (2018). Mindfulness meditation is related to long-lasting changes in hippocampal functional topology during resting state: a magnetoencephalography study. Neural plasticity, 2018. https://doi.org/10.1155/2018/5340717

[24] Jones, T. M. (2018). The effects of mindfulness meditation on emotion regulation, cognition and social skills. European Scientific Journal, 14(14), 18-32. https://doi.org/10.19044/ esj.2018.v14n14p18

[25] Brown, K. W., \& Ryan, R. M. (2003). The benefits of being present: mindfulness and its role in psychological well-being. Journal of personality and social psychology, 84(4), 822. https://doi.org/10.1037/0022-3514.84.4.822

[26] Mirams, L., Poliakoff, E., Brown, R. J., \& Lloyd, D. M. (2013). Brief body-scan meditation practice improves somatosensory perceptual decision making. Consciousness and Cognition, 22(1), 348-359. https://doi.org/10.1016/j.concog.2012.07.009

[27] Dorjee, D. (2016). Defining contemplative science: The metacognitive self-regulatory capacity of the mind, context of meditation practice and modes of existential awareness. Frontiers in psychology, 7, 1788. https://doi.org/10.31231/osf.io/9f65j

[28] Drigas, A., \& Karyotaki, M. (2017). Attentional control and other executive functions. International Journal of Emerging Technologies in Learning (iJET), 12(03), 219-233. https://doi.org/10.3991/ijet.v12i03.6587

[29] Hill, C. L., \& Updegraff, J. A. (2012). Mindfulness and its relationship to emotional regulation. Emotion, 12(1), 81.

[30] Jerath, R., Crawford, M. W., Barnes, V. A., \& Harden, K. (2015). Self-regulation of breathing as a primary treatment for anxiety. Applied psychophysiology and biofeedback, 40(2), 107-115. https://doi.org/10.1007/s10484-015-9279-8

[31] Clark, D., Schumann, F., \& Mostofsky, S. H. (2015). Mindful movement and skilled attention. Frontiers in Human Neuroscience, 9, 297. https://doi.org/10.3389/fnhum.2015.00297

[32] Yeung, A., Chan, J. S., Cheung, J. C., \& Zou, L. (2018). Qigong and Tai-Chi for mood regulation. Focus, 16(1), 40-47. https://doi.org/10.1176/appi.focus.20170042 
[33] Stanszus, L. S., Frank, P., \& Geiger, S. M. (2019). Healthy eating and sustainable nutrition through mindfulness? Mixed method results of a controlled intervention study. Appetite, 141, 104325. https://doi.org/10.1016/j.appet.2019.104325

[34] Rubin, R. D., Watson, P. D., Duff, M. C., \& Cohen, N. J. (2014). The role of the hippocampus in flexible cognition and social behavior. Frontiers in human neuroscience, 8, 742.

[35] Hölzel, B. K., Lazar, S. W., Gard, T., Schuman-Olivier, Z., Vago, D. R., \& Ott, U. (2011). How does mindfulness meditation work? Proposing mechanisms of action from a conceptual and neural perspective. Perspectives on psychological science, 6(6), 537-559. https://doi.org/10.1177/1745691611419671

[36] Drigas, A. S., \& Papoutsi, C. (2018). A new layered model on emotional intelligence. Behavioral Sciences, 8(5), 45.

[37] Mills, P. J., Barsotti, T. J., Blackstone, J., Chopra, D., \& Josipovic, Z. (2020). Nondual Awareness and the Whole Person. Global Advances in Health and Medicine, 9, 2164956120914600. https://doi.org/10.1177/2164956120914600

[38] Batchelor, S. (1997). Buddhism without beliefs: A contemporary guide to awakening. New York: Riverhead Books.

[39] Kang, C., \& Whittingham, K. (2010). Mindfulness: A dialogue between Buddhism and clinical psychology. Mindfulness, 1(3), 161-173. https://doi.org/10.1007/s12671-010$\underline{0018-1}$

[40] Vago, D. R., \& Zeidan, F. (2016). The brain on silent: mind wandering, mindful awareness, and states of mental tranquility. Annals of the New York Academy of Sciences, 1373(1), 96. https://doi.org/10.1111/nyas.13171

[41] MacLean, K. A., Ferrer, E., Aichele, S. R., Bridwell, D. A., Zanesco, A. P., Jacobs, T. L.\& Wallace, B. A. (2010). Intensive meditation training improves perceptual discrimination and sustained attention. Psychological science, 21(6), 829-839. https://doi.org/10.1177/ 0956797610371339

[42] Hofmann, S. G., Grossman, P., \& Hinton, D. E. (2011). Loving-kindness and compassion meditation: Potential for psychological interventions. Clinical psychology review, 31(7), 1126-1132. https://doi.org/10.1016/j.cpr.2011.07.003

[43] Lyles, M. A. (1994). An analysis of discrimination skills as a process of organizational learning. The Learning Organization.

[44] Krishnakumar, D., Hamblin, M. R., \& Lakshmanan, S. (2015). Meditation and yoga can modulate brain mechanisms that affect behavior and anxiety-A modern scientific perspective. Ancient science, 2(1), 13. https://doi.org/10.14259/as.v2i1.171

[45] Mahajan, A. S. (2014). Role of yoga in hormonal homeostasis. International Journal of Clinical and Experimental Physiology, 1(3), 173-178.

\section{Authors}

Athanasios Drigas is a Research Director at N.C.S.R. 'Demokritos', Institute of Informatics and Telecommunications - Net Media Lab \& Mind-Brain R\&D, Agia Paraskevi, 153 10, Athens, Greece (e-mail: dr@iit.demokritos.gr).

Eleni Mitsea is with Institute of Informatics and Telecommunications - Net Media Lab \& Mind-Brain R\&D, Agia Paraskevi, 153 10, Athens, Greece (e-mail: e.mitsea@gmail.com).

Article submitted 2020-07-31. Resubmitted 2020-10-16. Final acceptance 2020-10-17. Final version published as submitted by the authors. 